ORIGINAL ARTICLE

\title{
Efficient secretion of a modified E7 protein from human papilloma virus type-16 by Lactococcus lactis
}

\author{
D. Quistián-Martínez ${ }^{1}$, J. Villatoro-Hernández ${ }^{2}$, M.J. Loera-Arias², B.R. Rangel-Colmenero², \\ L.M. Zavala-Flores², J. Sepúlveda-Saavedra², S. Guzmán-López ${ }^{3}$, R.E. Elizondo-Omaña ${ }^{3}$, \\ R. Montes-de-Oca-Luna ${ }^{2}$ and O. Saucedo-Cárdenas ${ }^{2,4}$ \\ 1 Departamento de Bioquímica, Facultad de Ciencias Biológicas, Universidad Autónoma de Nuevo León (UANL), San Nicolás de los Garza, N.L. \\ México \\ 2 Departamento de Histología, Facultad de Medicina, UANL, Monterrey, N.L. México \\ 3 Departamento de Anatomía Humana, Facultad de Medicina, UANL, Monterrey, N.L. México \\ 4 División de Genética, Centro de Investigación Biomédica del Noreste, Instituto Mexicano del Seguro Social (IMSS), Monterrey, N.L. México
}

\section{Keywords}

antitumour protection, HPV-16, Lactococcus

lactis, modified E7, secretion.

\section{Correspondence}

Odila Saucedo-Cárdenas, División de Genética, Centro de Investigación Biomédica del Noreste, IMSS. Col. Independencia, Monterrey, N. L. México 64720.

E-mail: odilam@hotmail.com

D. Quistián-Martínez and J. VillatoroHernández have contributed equally to this work.

Present address: J. Villatoro-Hernandez, Molecular Genetics, Groningen Biomolecular Sciences and Biotechnology Institute, University of Groningen, Kerklaan 30, 9751 NN Haren, NED. Kluyver Centre for Genomics of Industrial Fermentation, PO Box 5057, 2600 GA Delft, The Netherlands.

2010/0493: received 26 March 2010, revised 2 July 2010 and accepted 11 July 2010

doi:10.1111/j.1472-765X.2010.02905.x

\begin{abstract}
Aims: To create and provide a strain of the food-grade bacterium Lactococcus lactis able to efficiently secrete a modified form of the E7 protein from the human papilloma virus (HPV) type-16.

Methods and Results: We cloned the coding sequence of a modified E7 (E7m) from the HPV-16 in a plasmid regulated by the strong expression promoter p59. Secretion of the E7m was made by the signal peptide of the usp45 gene. The E7m was detected by Western blot in the cell-free-medium fraction, showing no degradation or aberrant forms.

Conclusions: We constructed a strain of L. lactis able to secrete efficiently a HPV-16 E7 modified protein with diminished transforming activity.

Significance and Impact of the Study: Human papilloma virus infection is associated with more than $99 \%$ of cervical cancers. Immunotherapy targeting E7 to treat HPV-associated cervical malignancies has been demonstrated to be highly efficient. However, native E7 maintains transforming activity. We present this new strain of a food-grade bacterium able to efficiently secrete a HPV-16 E7-modified protein with diminished transforming activity. This new strain could be used as a live vaccine to deliver E7 at a mucosal level and generate antitumour immune responses against HPV-associated tumours.
\end{abstract}

\section{Introduction}

More than $99 \%$ of cervical cancers are associated with the human papilloma virus (HPV). In these HPV-associated tumours, two viral proteins remain expressed, E6 and E7. The development of vaccines and immunotherapies against cervical tumours targeting these viral oncoproteins has been documented to be effective and highly immunogenic (Roden and Wu 2006; Kim et al. 2008). Immunization with E7 has been shown to cause specific therapeutic effects against established E7-expressing tumours in mice
(Kim et al. 2007). Even though the use of this viral protein as immunotherapy against cervical cancer was shown to be specific and effective, it possesses an undesired characteristic. This unfavourable condition is that E7 maintains its transforming activity (Shi et al. 1999). This inconvenience was overcome by the modification of two repeating Cys-X-X-Cys sequence motifs at the carboxyl terminus of $\mathrm{E} 7$ in the $\mathrm{CR} 3$ domain containing residues 58-61 and 91-94 with repeating cysteines, which is necessary for the formation of a zinc finger, an important structure that gives stability to the E7 protein. The inability of 
this modified E7 to form this particular structure dramatically diminishes its transforming activity in cultures in vitro of murine and human cells (McIntyre et al. 1993).

It has been demonstrated that this modified E7 is degraded rapidly by the proteosome and that a greater amount of antigenic peptides are being transported to the endoplasmatic reticulum and presented to antigen-presented cells (APCs) (Shi et al. 1999). Moreover, this modified E7 with altered motifs has a reduced ability to transform a variety of rodent fibroblasts, fails to immortalize human keratinocytes, and exhibits a lower transactivating efficiency (McIntyre et al. 1993). Immunization with this modified E7 caused stronger protection immunity against E7-expressing cell lines than the wild-type E7 (Shi et al. 1999).

For years, the use of L. lactis to produce and deliver heterologous proteins of medical interest has been demonstrated to be efficient and innocuous (Wells and Mercenier 2008). The intranasal immunization with this food-grade bacterium expressing antigens has resulted in the generation of specific protective and therapeutic responses against infectious and chronic diseases (Hanniffy et al. 2007; Wells and Mercenier 2008). We have constructed a strain of the lactic acid bacterium (LAB) L. lactis able to secrete a modified E7 of the HPV that may be used as a live vaccine against the HPV-16 and tumours associated with this virus.

\section{Materials and methods}

\section{Bacterial strains and growth conditions}

Escherichia coli $\mathrm{DH} 5 \alpha$ was grown aerobically in Luria Bertani broth at $37^{\circ} \mathrm{C}$ with vigorous agitation. The L. lactis strain MG1363 was grown aerobically in M17 broth supplemented with $0.5 \%$ glucose (GM17) at $30^{\circ} \mathrm{C}$ without agitation. Selection of clones was carried out by the addition of $100 \mu \mathrm{g} \mathrm{ml}^{-1}$ of ampicillin for E. coli and $75 \mu \mathrm{g} \mathrm{ml}^{-1}$ of erythromycin for L. lactis. All constructions were first established in E. coli and then transferred to L. lactis by electrotransformation using the method previously described (Holo and Nes 1989). Plasmid DNA isolation, DNA manipulation, and PCR were carried out essentially as described (Sambrook et al. 1989).

\section{Protein extraction from $L$. lactis}

Cell and cell-free-medium fractions were prepared separately. Samples were processed from $1.35 \mathrm{ml}$ of the culture. Cell pellets were obtained by centrifugation at $21000 \mathrm{~g}$ at $4^{\circ} \mathrm{C}$ for $5 \mathrm{~min}$, and the pellets resuspended in $100 \mu \mathrm{l}$ of TES-lysis buffer (25\% sucrose, $1 \mathrm{mmol}^{-1}$ EDTA, $50 \mathrm{mmol} \mathrm{l}^{-1}$ Tris'HCL [pH 8.0], lysozyme [10 $\mathrm{mg} \mathrm{ml}^{-1}$ ] complemented with $1 \mathrm{mmol}^{-1}$ phenylmethylsulfonylfluoride (PMSF), and $10 \mathrm{mmol}^{-1}$ of dithiothreitol). The mixture was incubated at $37^{\circ} \mathrm{C}$ for one h, and then $50 \mu \mathrm{l}$ of $20 \%$ SDS and one volume of loading buffer were added. The samples were maintained at $-20^{\circ} \mathrm{C}$ before loading onto the gel.

The cell-free-medium samples were treated with $1 \mathrm{mmol} \mathrm{l}^{-1}$ of PMSF and $10 \mathrm{mmol} \mathrm{l}^{-1}$ of dithiothreitol to avoid proteolysis. Proteins were precipitated using $150 \mu \mathrm{l}$ of $100 \%$ trichloroacetic acid (TCA) and incubated on ice for $10 \mathrm{~min}$, then followed by centrifugation at $21000 \mathrm{~g}$ at $4^{\circ} \mathrm{C}$ for $15 \mathrm{~min}$. The pellet was resuspended in $50 \mu \mathrm{l}$ of $50 \mathrm{mmol}^{-1} \mathrm{NaOH}$ and $50 \mu \mathrm{l}$ of SDS-PAGE loading buffer (100 mmol l-1 Tris $\mathrm{HCl},[\mathrm{pH} 6 \cdot 8], 200 \mathrm{mmol} \mathrm{l}^{-1}$ dithiothreitol, $4 \%$ SDS, $0 \cdot 1 \%$ bromophenol blue, and $10 \%$ glycerol).

\section{SDS-PAGE and Western blotting}

One-fifteenth of the whole cell extracts and one-fifth of the cell-free-medium fractions from $1.35 \mathrm{ml}$ culture were loaded onto $12 \%$ acrylamide gels. SDS-PAGE and Western blotting were carried out essentially as described (Sambrook et al. 1989). Immunodetection of E7 was carried out by the use of monoclonal anti-E7 (Santa Cruz Biotechnology, Santa Cruz, CA) as a primary antibody and protein-G horseradish peroxidase conjugate (BioRad, Hercules, CA) and the SuperSignal West Pico Chemiluminescent Substrate kit (Thermo Scientific Pierce, Rockford, IL) as recommended by the suppliers.

\section{Coomassie Blue staining}

Twelve per cent SDS-PAGE gels with electrophoresed protein samples were submerged in CBB Buffer $(0 \cdot 1 \%$ Coomassie Brilliant Blue R-250, 20\% methanol, 0.5\% acetic acid) overnight. The gel was incubated in destaining buffer (40\% methanol, $7 \%$ acetic acid) for $2 \mathrm{~h}$ at room temperature with mild agitation.

\section{Results}

Generation of an expression system to secrete the modified E7 by L. lactis

The modified-E7 DNA coding sequence was PCR-amplified from plasmid BC219-E7 (kindly donated by Dr Liang Qiao) using oligos SalI-Fw 5-AGTCGACCCATTGCATG GAGATACACCTACATTG-3 and TER-Rv 5-ATCAAAA AAAAGCCCGCTCATTAGGCGGGCTATTTATGGTTTC TGAGAACAGA-3. SalI-Fw oligo generated a SalI restriction site in the E7 amplicon (underlined) at the $5^{\prime}$ termini that allowed the cloning of the $\mathrm{E} 7 \mathrm{~m}$ sequence 
in-frame with the signal peptide of usp45, the most secreted protein of L. lactis (van Asseldonk et al. 1990). The TER-Rv oligo generated a rho-independent transcription terminator for clone stability (underlined) and a stop codon (in bold). Amplicons were directly cloned into pGEM-T Easy Vector (Promega, Madison, WI) and then digested with SalI-SpeI sequentially. The fragment corresponding to E7m was subcloned in the pVE-5524 vector cut with the same enzymes. This new plasmid (pSECE7m) has controlling sequences to allow expression and secretion of E7m under the regulation of the high expression and constitutively active promoter p59 (Fig. 1). The pSEC-E7m was introduced into the L. lactis strain MG1363 by electroporation. Clones were selected by the resistance to erythromycin. This newly constructed strain of L. lactis was named Ll-pSEC-E7m. All constructs were characterized by endonuclease restriction analysis and PCR and sequenced to verify DNA integrity.

\section{Secretion of E7m by L. lactis}

Detection of E7m by Coomassie blue staining. To evaluate the ability of the strain Ll-pSEC-E7m to secrete E7m, protein extracts from cell and cell-free-medium fractions were made from cultures of our recombinant strain. For this, we allowed cultures of the Ll-pSEC-E7m to reach an $\mathrm{OD}_{600 \mathrm{~nm}}$ of one. From each culture, $1.35 \mathrm{ml}$ was centrifuged at $17000 \mathrm{~g}$ for five $\min$ at $4^{\circ} \mathrm{C}$ to separate bacteria and the cell-free medium. The cell-free medium was filtered through a $0 \cdot 22-\mu \mathrm{m}$ pore-diameter (Milipore, Billerica, MA) filter to remove the remaining bacteria. The total proteins were precipitated with TCA (for more details, see materials and methods). Samples from the cell fractions of L. lactis were prepared as well. After electrophoresis, acrylamide gels were stained with Coomassie Blue to visualize total proteins from the cell- and cellfree-medium protein extracts from cultures of L. lactis.

Figure $2 \mathrm{a}$ shows the electrophoresed L. lactis protein samples from the strains transformed with an empty vector (pSEC) and our recombinant strain Ll-pSEC-E7m. Samples from the cell-free medium of our recombinant

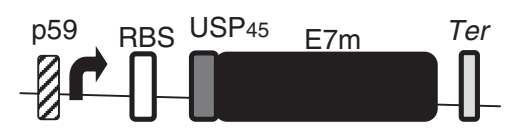

Figure 1 Expression cassette for E7m secretion by L. lactis. Coding sequence of E7m was placed under control of the high-expression constitutive p59 promoter (stripped bar), the ribosome binding site (RBS) indicated in the empty bar, and the signal peptide of usp45 from L. lactis. The grey bar (Ter) represents a rho-independent trpA transcription terminator fused just downstream to the E7m gene. strain revealed the secretion of a distinctive protein band that corresponds to the expected size of the E7m (indicated with asterisk in Fig. 2a). This particular protein band is absent in the cell-free-medium samples from L. lactis transformed with the empty vector pSEC (lacking the E7m-coding sequence) and in whole cell-extract samples from both strains. The stain intensity of this secreted protein is close in amount to the major lactococcal secreted protein Usp45 (indicated by a double asterisk in Fig. 2a).

Detection of E7m by Western blot. To determine whether the secreted protein band seen in the SDS-PAGE protein gel corresponds to the protein E7, a duplicated gel from Fig. 2a was analysed by immunoblotting using a mouse monoclonal anti-E7 (Santa Cruz). Western blot analysis revealed a single clear band in the sample from the cellfree cultures of our recombinant strain Ll-pSEC-E7m corresponding to the expected size of E7m (Figure 2b). Moreover, no signal was detected in the whole cell fraction of this recombinant strain, meaning that the secretion of E7m was achieved successfully and that no protein remained inside the cell. As expected, no signal was detected in either of the samples from cell-free cultures or cell fractions of L. lactis wild-type (data not shown) or in L. lactis transformed with an empty vector (pSEC) with no E7m coding sequence (Fig. 2b).

\section{Discussion}

The E7 protein of the HPV has been demonstrated to be a good target to develop both prophylactic and therapeutic tumour vaccines (Roden and $\mathrm{Wu}$ 2006; Kim et al. 2008). Immunization with this antigen has been demonstrated to cause a specific, cytotoxic immune response against tumours expressing E7. An adverse side effect of this is that E7 maintains its transforming activity, increasing the risk of cellular transformation.

We made a strain of the food-grade bacterium L. lactis, a lactic acid bacterium (LAB), able to secrete a modified E7 from the HPV type-16. To address the E7m outside the cell, we made use of the signal peptide from the usp45 protein, which is the most secreted protein of L. lactis (van Asseldonk et al. 1993).

Western blot analysis using specific monoclonal antibodies corroborated the highly efficient secretion of E7m by L. lactis. Only one strong signal corresponding to E7m was detected in the cell-free-medium sample of the recombinant lactococcus. The absence of signal in the whole-cell extract sample means that the E7m protein was efficiently processed and secreted by this recombinant lactococcus. 

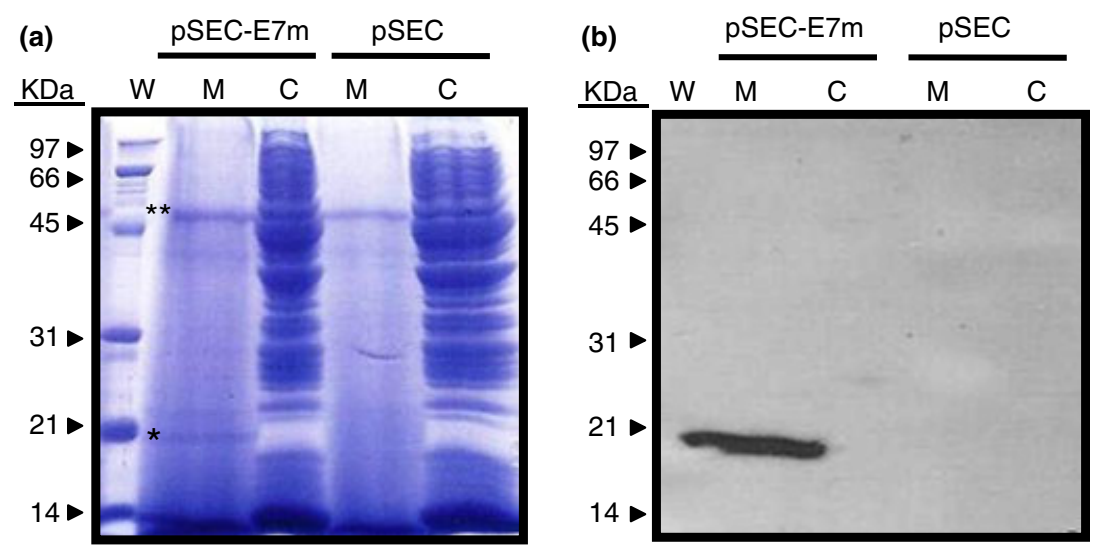

Figure 2 Expression analysis of E7m secretion by recombinant L. lactis. The cell fraction (C) and cell-free-medium (M) proteins from cultures of L. lactis were electrophoresed by SDS-PAGE and analysed by Coomassie Blue staining (2a) and Western blotting using specific anti-E7 antibodies (2b). An 18-kDa band of the expected size $\left(^{*}\right)$ was detected in the recombinant strain carrying the plasmid pSEC-E7m. W is the molecular mass marker and $\left(^{* *}\right)$ indicates the native usp45 protein. Only an 18-kDa band corresponding to E7m was detected in the recombinant strain carrying the plasmid pSEC-E7m in samples from the cell-free medium. No signal was detected in the cell fraction of this same strain or in the control strain transformed with the mock vector.

The secreted protein identified as E7 by Western blot corresponds to the Coomassie-Blue-stained protein shown in the SDS-PAGE gels. It is noteworthy to compare the intensity of this E7m band (indicated with an asterisk in Fig. 2a) with that of the usp45, which is the major protein secreted in L. lactis (indicated with a double asterisk). In this work, we used the usp45 secretion signal to secrete our E7m. Both the usp45-protein bands, from strains transformed with an empty vector (with no E7m coding DNA sequence) and the recombinant transformed with the vector coding for E7m, were similar in intensity. This outcome indicates how a heterologous protein expressed by $L$. lactis using an endogenous-signal peptide may be secreted in the same manner and undergo the same processing as the native protein from which the signal peptide was used.

Because this strain of L. lactis is able to secrete considerable amounts of $\mathrm{E} 7 \mathrm{~m}$, it could be used in large-scale bioreactors to produce $\mathrm{E} 7 \mathrm{~m}$ in large amounts, with several benefits including the favourable quality of this food-grade bacterium that does not produce lipopolysaccharides or any other toxins.

For years, L. lactis has been used to express heterologous proteins of medical importance. In 2008, the intranasal immunization with a strain of L. lactis expressing the PspA antigen of Streptococcus pneumoniae demonstrated the ability to generate a protective immunity in mice challenged with this pathogen (Hanniffy et al. 2007). Another strain of L. lactis expressing the interleukin 10 is now in Phase I of clinical trials in humans to treat the inflammatory bowel disorder Crohn's disease (Braat et al. 2006). Because the use of this micro-organism has been shown to be competent and safe, and more importantly, to cause specific immune responses against chronic and infectious diseases, we propose the use of this new strain of L. lactis able to secrete the E7 of the HPV for the development of a new live vaccine against cervical cancer. Further studies will be necessary to confirm the diminished transforming properties of this modified E7 antigen to cause specific antitumour activity against HPV-associated tumours and infections with this virus associated in more than $90 \%$ of cervical cancers.

Actogenix and Mucosis (to our knowledge) are the first two companies offering several vaccines based on L. lactis expressing immonomodulators and antigens of viral and bacterial origin to treat pneumonia, mucositis, and influenza. The value of more strains available to make preclinical and clinical trials using L. lactis can provide feasible and imminent new biopharmaceuticals that can become commercially available in a short time. This strain, efficiently secreting E7, may be able to determine the therapeutic ability to cause specific immune responses against E7-tumour-bearing organisms, without the transforming activity of E7 (Audouy et al. 2007; Steidler et al. 2009).

\section{Acknowledgements}

JVH, MJLA, BRRC and LMZF are recipients of a PhD scholarship from CONACyT of Mexico. The authors thank Dr Qiao for donating BC219-E7 plasmid. Thanks to Dr Ellis Glazier for editing this English-language text.

\section{References}

van Asseldonk, M., Rutten, G., Oteman, M., Siezen, R.J., de Vos, W.M. and Simons, G. (1990) Cloning of usp45, a 
gene encoding a secreted protein from Lactococcus lactis subsp. lactis MG1363. gene 95, 155-160.

van Asseldonk, M., de Vos, W.M. and Simons, G. (1993) Functional analysis of the Lactococcus lactis usp45 secretion signal in the secretion of a homologous proteinase and a heterologous alpha-amylase. Mol Gen Genet 240, 428-434.

Audouy, S.A., van Selm, S., van Roosmalen, M.L., Post, E., Kanninga, R., Neef, J., Estevão, S., Nieuwenhuis, E.E. et al. (2007) Development of lactococcal GEM-based pneumococcal vaccines. Vaccine 25(13), 2497-2506.

Braat, H., Rottiers, P., Hommes, D.W., Huyghebaert, N., Remaut, E., Remon, J.P., van Deventer, S.J., Neirynck, S. et al. (2006) A phase I trial with transgenic bacteria expressing interleukin-10 in Crohn's disease. Clin Gastroenterol Hepatol 4, 754-759.

Hanniffy, S.B., Carter, A.T., Hitchin, E. and Wells, J.M. (2007) Mucosal delivery of a pneumococcal vaccine using Lactococcus lactis affords protection against respiratory infection. J Infect Dis 195, 185-193.

Holo, H. and Nes, I.F. (1989) High-Frequency Transformation, by Electroporation, of Lactococcus lactis subsp. cremoris Grown with Glycine in Osmotically Stabilized Media. Appl Environ Microbiol 55, 3119-3123.

Kim, D., Hoory, T., Wu, T.C. and Hung, C.F. (2007) Enhancing DNA vaccine potency by combining a strategy to prolong dendritic cell life and intracellular targeting strategies with a strategy to boost CD4 $+\mathrm{T}$ cell. Hum Gene Ther 18, 1129-1139.

Kim, D., Gambhira, R., Karanam, B., Monie, A., Hung, C.F., Roden, R. and Wu, T.C. (2008) Generation and characterization of a preventive and therapeutic HPV DNA vaccine. vaccine 26, 351-360.

McIntyre, M.C., Frattini, M.G., Grossman, S.R. and Laimins, L.A. (1993) Human papillomavirus type 18 E7 protein requires intact Cys-X-X-Cys motifs for zinc binding, dimerization, and transformation but not for Rb binding. J Virol 67, 3142-3150.

Roden, R. and Wu, T.C. (2006) How will HPV vaccines affect cervical cancer? Nat Rev Cancer 6, 753-763.

Sambrook, J., Fritsch, E.F. and Maniatis, T. (1989). Molecular Cloning: A Laboratory Manual, 2nd edn. NY.

Shi, W., Bu, P., Liu, J., Polack, A., Fisher, S. and Qiao, L. (1999) Human papillomavirus type 16 E7 DNA vaccine: mutation in the open reading frame of E7 enhances specific cytotoxic T-lymphocyte induction and antitumour activity. J Virol 73, 7877-7881.

Steidler, L., Rottiers, P. and Coulie, B. (2009) Actobiotics as a novel method for cytokine delivery. Ann N Y Acad Sci 1182, 135-145.

Wells, J.M. and Mercenier, A. (2008) Mucosal delivery of therapeutic and prophylactic molecules using lactic acid bacteria. Nat Rev Microbiol 6, 349-362. 